Stoa

Vol. 3, no. 5, 2012, pp. 13-35

ISSN 2007-1868

\title{
LA DISTINCIÓN ENTRE TÉRMINOS T-TEÓRICOS Y T-NO-TEÓRICOS. COMPROMISOS ONTOSEMÁNTICOS
}

\author{
Adriana Gonzalo \\ FHUC/FADU \\ Universidad Nacional del Litoral \\ adriana.n.gonzalo@gmail.com
}

RESUMEN: En la primera parte se introduce el criterio de teoreticidad de Sneed (1971) y posteriormente las propuestas de Stegmüller (1973, 1976) y Balzer y Moulines (1980, 1985), considerando a todas ellas como perspectivas "pragmáticas". A continuación se presenta la perspectiva de Balzer (1986, 1996) y se le inscribe dentro de las perspectivas "semánticas" de la problemática.

En la segunda parte, el trabajo se centra en el problema de los compromisos ontosemánticos que acarrea la distinción entre términos $T$-teóricos y lo $T$-no-teóricos; en el marco de las propuestas pragmáticas, particularmente la de Moulines (1982, 1984, 1991, 1994, 1998, 2002). Se realiza una evaluación de la misma, señalando en qué aspectos representa ésta una innovación importante en la elucidación metateórica de la ontosemántica de las teorías científicas, y en qué otros presenta limitaciones.

PALABRAS CLAVE: teorías científicas · concepción estructural · términos teóricos criterios de teoreticidad · ontosemántica

ABST RACT: First, Sneed's criterion of theoreticity is introduced (Sneed 1971) and then the proposals of Stegmüller (1973, 1976), Balzer and Moulines $(1980,1985)$ are presented. All of them are considered as "pragmatic" views of theoreticity. Afterwards, the perspective of Balzer $(1986,1996)$ is offered and included in the "semantic"view of the problem.

Secondly, the work focuses on the problem of ontosemantic commitments that result from the distinction between $T$-theoretical and $T$-non-theoretical terms from the perspective of pragmatic proposals, taking into account Moulines' position $(1982,1984,1991,1994,1998,2002)$ on this topic. An evaluation 
of his position is carried out, pointing out the aspects in which it represents an important innovation in the metatheoretical elucidation of the ontosemantic of scientific theories, and those which present limitations.

KeYwords: Scientific theories - structuralist view of theories - theoretical terms - criteria of theoreticity $\cdot$ ontosemantic

\section{Introducción}

\section{1}

El presente trabajo se enmarca en el conjunto de reflexiones, dentro de la concepción estructuralista de la ciencia $(\mathrm{CE})$, en relación a una agenda de los principales problemas ontosemánticos de las teorías científicas. Partiré de considerar que estos problemas se configuran sobre los siguientes ejes:

a) Distinción entre términos teóricos relativos a una teoría $T$ (términos $T$-teóricos) y no teóricos, relativos a $T$ ( $T$-no-teóricos), en contraposición a la distinción global de la visualización estándar de conceptos observacionales y conceptos teóricos.

b) La distinción supone una diferenciación de base entre modelos (reales) y modelos (potenciales parciales) de una teoría (elemento teórico) en cuestión.

c) La noción de "aplicaciones intencionales" de una teoría, partiendo de que éstas se conciben como un subconjunto de los modelos potenciales parciales de una teoría.

d) La determinación de los aspectos semánticos y pragmáticos que permean la distinción de los términos en las teorías científicas.

e) Los aspectos ontológicos que subyacen a la distinción entre $T$-teórico, $T$-no-teórico y el modo en que éstos afectan una posición ontológica general de la ciencia.

f) El problema de las aserciones empíricas de una teoría, y su justificación, que involucran términos epistemológicos como "dar sustento", "confirmar", etcétera, que también ponen en discusión lo que entendemos por "componentes extralingüísticos" de las teorías. 
g) La distinción entre aspectos sincrónicos y diacrónicos que son relativos a la determinación del significado de los términos en las teorías científicas y cambio de significado.

Claramente, este vasto conjunto de problemas no puede tratarse con profundidad en un trabajo de esta naturaleza, de modo que me limitaré a trazar una estrategia que me permita concentrarme en el problema de la distinción entre términos $T$-teóricos y $T$-no-teóricos, y los compromisos ontológicos que la misma conlleva.

En la primera parte del trabajo presentaré la versión originaria de la problemática y la solución presentada en Sneed (1971), además de comentar brevemente algunos desarrollos posteriores realizados por Stegmüller $(1973,1976)$ que tuvieron continuidad en Balzer y Moulines (1980) y Moulines (1985), deteniéndome particularmente en este último. Caracterizaré estas soluciones como propuestas "pragmáticas".

Completando una presentación esquemática del problema antes aludido, mencionaré otros desarrollos de la distinción $T$-teórico, $T$ no-teórico en Balzer (1986) y Balzer (1996). Estos trabajos se inscriben en lo que denominaré propuestas "semánticas" de la problemática.

En la segunda parte, el trabajo se centrará en el problema de los compromisos ontosemánticos que acarrea esta distinción en el marco de las propuestas pragmáticas, particularmente en la posición de Moulines (1982, 1984, 1991, 1994, 1998, 2002). Caracterizaré su posición como: (a) una defensa de la tesis de la determinación primaria del lenguaje en el modo de acceder a los entes en general y a las entidades científicas en particular; (b) una salida pragmática circunscripta a la pragmática del significado (en contraposición a lo que podría ser una posición pragmática relativa a un pragmatismo o una posición praxista).

Finalmente realizaré una evaluación de la propuesta metateórica de Moulines en las obras citadas, señalando en qué aspectos representa ésta una innovación importante en la elucidación metateórica sobre la ontosemántica de las teorías científicas y en qué otros adolece de problemas, presentando ciertos límites en la solución ofrecida. 


\section{Primera Parte}

\section{2}

En la concepción sneediana (Sneed 1971) se caracterizaba por primera vez un criterio de teoreticidad que permitía una distinción entre términos $T$-teóricos y $T$-no-teóricos, a la vez que brindaba una respuesta a lo que Stegmüller (1973) haría popular como "el reto de Putnam", esto es, la respuesta a la cuestión sobre qué son los términos teóricos en la ciencia.

Para que esta distinción sea comprendida debemos hacer algunas consideraciones previas. En la CE se han dado dos definiciones precisas de "teoría": según una caracterización restringida, teoría se define como "elemento teórico"; mientras que en un sentido más amplio, teoría se define como red-teórica. ${ }^{1}$ La caracterización de Sneed (1971) se relaciona con la noción restringida de teoría. Partiendo de ella introduciré un marco de nociones básicas del planteamiento sneediano que permitirán la comprensión de este criterio. ${ }^{2}$

En la obra arriba mencionada, Sneed presentaba una concepción de "teoría" - pensada particularmente para las teorías físicas- y se expresaba formalmente mediante la dupla $\langle K, I\rangle$, donde $K$ representa la estructura formal matemática de la teoría e $I$ el conjunto de aplicaciones intencionales (intended applications). Sneed pensaba que la forma más adecuada de conceptualizar $K$ consistía en concebirla como un conjunto de modelos que constituyeran las unidades de análisis de las teorías. Así se introdujo esta noción, definida como un procedimiento de axiomatización por la introducción de un predicado conjuntista. Esta axiomatización requería la diferenciación entre modelo potencial $\left(M_{p}\right)$ y modelo actual $(M)$ de una teoría (física). Se concibió $M_{p}$ como una entidad determinada por las condiciones estructurales que intervienen en la caracterización del predicado conjuntista correspondiente a la axiomatización de la teoría en cuestión; es decir, $M_{p}$ es cualquier

\footnotetext{
${ }^{1}$ Una caracterización precisa de estas nociones se da en Balzer, Moulines y Sneed (1987). La noción de "elemento teórico" se presenta en el cap. II, mientras que la de "red-teórica" se desarrolla en el cap. IV. La caracterización de "teoría" en el sentido de "elemento-teórico" que se introducirá a continuación se remonta a la etapa fundacional de CE y es un tanto menos precisa y formal que la dada en la obra arriba indicada.

${ }^{2}$ Esta parte del texto está dirigido a un lector que no está muy familiarizado con las nociones centrales de la CE y tiene el objetivo de facilitarle la comprensión del planteo sneediano.
} 
sistema del que sabemos que tiene la estructura conceptual requerida para ser un modelo actual de $T$ mientras que, si añadimos un conjunto de axiomas o leyes generales de $T$ a las condiciones que determinan los $M_{p}$ de $T$, las entidades que satisfacen éstos serán denominadas modelos actuales de $T$. A esta diferenciación se sumó la idea de constraints $(C)$.

Sneed pensó además que una teoría tendría muchos modelos y que los mismos deberían estar interconectados formalmente conformando una estructura global. Así se propuso esta idea para incorporar al formalismo caracterizador de una teoría las constraints entre las funciones correspondientes a los diversos modelos de una teoría, además, a esto se sumó la idea de modelos potenciales parciales $\left(M_{p p}\right)$ de una teoría $T$ como estructuras que describen los sistemas (físicos) posibles a los que es concebible aplicar $T$.

La estructura formal de una teoría quedaba simbolizada así:

$$
K=\left\langle M_{p}, M, M_{p p}, C\right\rangle .
$$

Es precisamente este núcleo matemático formal el que describe el predicado conjuntista que axiomatiza una teoría y es justamente este predicado el que se utiliza para realizar aserciones empíricas de la teoría. Para abordar la comprensión de esta última noción, Sneed usará una idea característica de la física matemática: "applications". Las aserciones afirman que el predicado conjuntista se aplica sobre sistemas físicos que conforman el conjunto de las aplicaciones propuestas, características de la teoría. Para que esto sea posible es necesario que se haya conceptualizado estos sistemas, a los que se desea aplicar el formalismo del núcleo en términos compatibles con la conceptualización presentada en éste. Así, se concibió que estos sistemas debieran considerarse como estructuras del tipo de los modelos potenciales parciales de la teoría; es decir, estructuras que son compatibles con la teoría pero que no la presuponen. Por ende, se definió el conjunto de las aplicaciones propuestas de una teoría -que designamos con $I-$ como incluido en el conjunto de los $M_{p p} .^{3}$

Para nuestros propósitos nos interesa añadir que los $M_{p p}$ de una teoría $T$ eran concebidos como lo que podría llamarse la "base empíri-

\footnotetext{
${ }^{3}$ Desarrollos posteriores de la CE precisarán que $I \subseteq \operatorname{pot}\left(M_{p p} T\right)$ (Balzer, Moulines y Sneed
} (1987)). 
ca" de $T$. Este conjunto de entidades quedaba determinado por un predicado conjuntista definido por los mismos axiomas estructurales que determinan $M_{p}$, a excepción de aquellos que se refieren a los términos teóricos relativos a $T$. Debemos resaltar el aspecto de que $\operatorname{los} M_{p p}(T)$ son sistemas que se han "podado" de los términos teóricos relativos a $T$ y son caracterizables mediante otros términos pertenecientes a teorías diferentes de $T$. Adelantemos que este aspecto nos permitirá captar el enfoque ontológico de la CE. Por su parte, las aplicaciones intencionales de $T$, concebidas como subconjuntos de los $M_{p p}(T)$ constituye un modo original de presentar la idea de sistemas físicos que se caracterizan como sistemas conceptualizables en términos no pertenecientes a $T$, pero que pueden ser "modelables" mediante el aparato conceptual de $T$, resultando "aplicaciones exitosas" del mismo.

Al mismo tiempo Sneed había introducido la noción de "disponer de una teoría" en el sentido de que en un período histórico de desarrollo de ésta se dispone de un conjunto determinado de aplicaciones paradigmáticas del núcleo, que forman parte del conjunto total de aplicaciones del mismo sobre la base del cuál se producen los futuros cambios en dicho conjunto. Así, señalaba el carácter intencional de las aplicaciones y mostraba correlativamente el aspecto pragmático involucrado, esto al aludir a las actitudes de los científicos que disponen de una teoría $T$ en un tiempo $t$ con el que operan en sus prácticas científicas.

A partir de las nociones dadas podemos introducir el criterio de teoreticidad ( $\left.C T^{\prime}\right)$ de Sneed: un término $t_{i}$ de $T$ es $T$-teórico si en todas las determinaciones de $t_{i}$ las partes de la teoría usada (presupuesta) por los científicos con el propósito de su determinación son parte o coinciden con T; en otras palabras, los términos $T$-teóricos son términos que pueden ser determinados únicamente por medio de $T$.

Balzer (1996) ha hecho notar algunos problemas derivados de la propuesta de Sneed, según él, al proponer que los términos $T$-noteóricos componen los "modelos potenciales parciales", entre los cuales encontramos "aplicaciones intencionales", se corren ciertos riesgos prácticos, ya que hay teorías para las cuales es muy difícil encontrar cuáles de sus términos son teóricos. Balzer sostiene que se dan casos extremos en los que todos o ninguno de los términos son $T$-teóricos 
en la interpretación de la aserción empírica de Sneed: "Todas las aplicaciones intencionales pueden ser extendidas a modelos reales". Si todos los términos son $T$-teóricos esta aserción empírica se reduce a un enunciado sobre la cardinalidad de los conjuntos de base, si ningún término es $T$-teórico entonces el rango completo de los términos tiene que ser determinado o medido para el rango completo de los argumentos, antes de que la aserción empírica se compruebe (Balzer 1996, p. 154).

Balzer (1986) ha enfatizado que la idea que está en la base del CT de Sneed es una intuición sobre la práctica científica, se trata más de un criterio pragmático que de uno lógico. Para penetrar la significación del criterio debemos precisar dos expresiones usadas en su formulación original, esto es: "determinación de un término" y "presuposición de $T$ " en una determinación de un término.

En este texto de Balzer se ha hecho notar que, informalmente expresada, la idea central que subyace a las expresiones antes mencionadas parte de considerar la existencia de una teoría dada $T$ y la de un grupo de científicos que operan con $T$. Por alguna razón u otra se precisa conocer algunos valores de ciertas funciones de $T$ para determinados argumentos dados. Este conocimiento no puede adquirirse por "observación directa", esto al menos en el caso de teorías avanzadas, de modo que se requieren otras acciones: experimentos y mediciones que se llevan a cabo en orden a determinar los valores deseados. Todas estas actividades, que dan como resultado uno o más valores de las funciones identificables, representan las determinaciones para algún término. Teniendo en cuenta ejemplos concretos de tales determinaciones reconocemos que en el curso de una determinación los científicos en general usan conocimiento teórico, fórmulas o ecuaciones. Éstos realizan ciertos cálculos e inferencias sobre la base de fórmulas dadas a modo de obtener valores de la función que se desea conocer. Usualmente este conocimiento teórico se da en un contexto concreto sin mayor justificación: es (hipotéticamente) asumido o presupuesto, todos los tipos de suposiciones teóricas usadas en el curso de una determinación son presuposiciones. Para cualquier "porción de una presuposición" (una ecuación, una fórmula, etc.) hay dos casos posibles: o se da que provie- 
ne (es parte de) alguna teorías $T$ diferente de $T$ o es idéntica a $T$ (vale decir, coincide con los axiomas de T) (Balzer 1986, p. 74). La noción de "disponer de una teoría" fue desarrollada por Stegmüller (1975, 1976, entre otros). Stegmüller (1976, pp. 222-223) afirmaba que para la caracterización de la expresión se requería de conceptos extralógicos como "persona", "creer que", "tener evidencia de apoyo para", así como una variable temporal $t$. Estos conceptos se enmarcaban en el terreno de las actitudes proposicionales de sujetos pertenecientes a una comunidad científica en un tiempo $t$ determinado, de modo que "la persona $p$ cree que $Y$ " y " $p$ tiene evidencia empírica a favor de $Y$ " en un tiempo $t .{ }^{4}$ Según el autor estas nociones escapaban a una precisión formal y requerían de un tratamiento pragmático.

Por otra parte, se ha aseverado que Stegmüller (1973) interpretó la idea de Sneed de "presuposición" en términos de "implicación lógica", y concibió que un término $t_{i}$ es $T$-teórico si para toda determinación de (una realización de) $t_{i}$ las oraciones que describen las determinaciones de $t_{i}$ de acuerdo a cada exposición existente de $T$ implican lógicamente la existencia de alguna aplicación exitosa de $T$; es decir, algún sistema que satisface las leyes de $T .^{5}$

\section{4}

Posteriormente Balzer y Sneed (1977-78) se propusieron definir el núcleo matemático de un elemento teórico como:

$$
K=\left\langle M, M_{p}, M_{p p}, C, L\right\rangle,
$$

donde $L$ simboliza links interteóricos (vínculos interteóricos). Continuando el camino de búsqueda de una caracterización formal más adecuada, Balzer, W. y Moulines C.U. (1980) precisaron la noción de "en toda determinación" que aparecía en la formulación de Stegmüller (1973). Con este fin se complejizó el aparato teórico para explicar más claramente la noción de "determinación". ${ }^{6}$

\footnotetext{
${ }^{4}$ La noción de "disponer de una teoría” había sido sugerida ya por Sneed 1971 en relación a las nociones kuhnianas de comunidad científica y de ciencia normal. Este enfoque se desarrolló más ampliamente por Stegmüller 1973 y 1976, entre otros).

${ }^{5}$ Balzer 1996, p. 141.

${ }^{6}$ Posteriormente, Balzer, Moulines y Sneed 1987 caracterizaron el aparato formal de una teoría mediante:
}

$$
K=\left\langle M_{p}(T), M(T), M_{p p}(T), R G(T), V G(T)\right\rangle,
$$


A partir de esta conceptualización se enfatizó que para reconstruir formalmente una teoría científica $T$ se necesitan dos tipos de principios: los axiomas o principios internos para fijar la clase de los modelos de $T$, con la cual a su vez se identifica a $T$, y los principios puente para fijar los vínculos $(V)$ que $T$ tiene con otras teorías o links interteóricos. Los principios de la primera clase son los que se asocian con $M_{p}(T)$ y con $M(T)$, los de la segunda son aquellos que se asocian con algunas relaciones $V_{i}$ sobre $M_{p}(T) \times M_{p}\left(T_{i}\right)$.

En la visión estructuralista la existencia de los principios puente (vínculos interteóricos), y su clara diferenciación con respecto a los axiomas, juega un papel en la distinción entre términos teóricos y noteóricos. Así, en un marco de definición más preciso, Moulines (1985) ${ }^{7}$ presenta un criterio de teoreticidad modificado del siguiente modo:

Sea $f$ un término fundamental en $T$ (esto es, $f$ no puede definirse por medio de otros términos de $T$ ). Si se supone que $T$ es una teoría científica, entonces la determinación de la extensión de $f$ en $T$ no será arbitraria (...), sino que se seguirán algunos patrones de determinación especificables, intersubjetivos y repetibles. Cuando $f$ es un concepto métrico esos patrones son lo que normalmente se llama "métodos de medición". Con el fin de incluir el caso más general, en donde no necesariamente están involucrados conceptos métricos, hablamos de "métodos de determinación". Estos corresponden a cosas tales como dispositivos de medición particulares junto con ciertas reglas para operarlos. Si queremos decir que estamos usando ciertos métodos para determinar $f$ dentro de $\mathrm{T}$, entonces las estructuras conceptuales que representan a esos métodos serán de un tipo semejante a, o compatible con, el tipo característico de $T$, de otra manera no tendríamos ninguna garantía para decir que estamos midiendo (o determinando) a $f$ dentro de $T$. En el caso más simple, las estructuras que representan a esos métodos de determinación de $f$ en $T$ coincidirían completamente con las estructuras características de la estructura conceptual de $T$, es decir, serán modelos potenciales de $T$. Si se denomina $M_{f}(T)$ al conjunto de todos los modelos potenciales de $T$ que se usan como métodos para determinar $f$, entonces la idea original de Sneed puede hacerse

donde $M_{p}(T)$ es la clase de los modelos potenciales de la teoría, $M(T)$ es la clase de los modelos de la teoría, $M_{p p}(T)$ es la clase de los modelos potenciales parciales de $T, R G(T)$ es la clase de las restricciones (global constraints) que caracterizan conexiones o relaciones entre diferentes aplicaciones o modelos de una misma teoría y $V G(T)$ es la clase de los vínculos interteóricos globales (global links) que caracterizan las conexiones esenciales de los elementos teóricos con otras teorías -representados por otros elementos teóricos.

${ }^{7}$ En la misma línea proseguirían los trabajos de Moulines (1992, 1998). 
más precisa por medio del siguiente "criterio de teoreticidad" $\left(C T^{\prime}\right): f$ es $T$ - teórico si $M_{f}(T) \subseteq M(T)$.

Ahora bien, parafraseando a Moulines, como las teorías no son entidades que se autocomprueben, no todos sus términos son $T$-teóricos. En una teoría científica $T$ siempre encontraremos algún término $g$ para el cual hay algún método de determinación dentro de $T$ que no es un modelo de $T$. Llamaremos $T$-no-teóricos a tales términos (Moulines 1985, p. 474).

Moulines agrega que los métodos de determinación de los términos $T$-no-teóricos de $T$ que no son modelos actuales de $T$ son los que permiten someter la teoría a una prueba independiente. [...] Proporcionan lo que normalmente se llama "los datos empíricos" para T. Si se simboliza a uno de tales métodos para determinar a un término $g$ $T$-no-teórico por " $n g$ ", se tiene que $n g$ determina la extensión de $g$ -si $g$ es una función métrica, $n g$ "mide" $g-$. Es aceptable considerar que $n g \in M_{p}(T)$, pero también tenemos que $n g \notin M(T)$; esto es, en este caso sólo los axiomas estructurales de $T$ pueden jugar un papel en la determinación de $g$, pero las leyes fundamentales y especiales de $T$ no juegan ningún papel. Puesto que se ha admitido que los axiomas estructurales son muy pobres en contenido, ¿̇cómo podemos suponer que $g$ puede determinarse por medio de $n g$ ? (Moulines 1985, p. 475). Es aquí donde intervienen -según el autor- los vínculos interteóricos. La información adicional que fija que $n g$ determine $g$ no es una cuestión de observación sino que intervendrá también información teórica, pero ésta será proveniente de alguna otra teoría a través de un vínculo apropiado, mismo que conecta al método de determinación de $g$ en $T$ con algún método de determinación de $g$ en $T$ para alguna otra función en alguna $T_{i}$ y este método de determinación $T_{i}$ presuntamente incluso satisface las leyes fundamentales de $T_{i}$ o también algunas de sus leyes especiales.

Así, según Moulines, es posible ligar la distinción entre términos teóricos y no-teóricos, por un lado, con la distinción entre axiomas, y con los principios puente por el otro, todo esto de la siguiente manera: los axiomas de la teoría (especialmente las leyes fundamentales) son prácticamente esenciales para determinar los términos teóricos; los principios puente son prácticamente esenciales para determinar los términos no-teóricos. 
Un problema que Sneed había dejado abierto es el de si todas las teorías científicas constan de términos $T$-no-teóricos. La propuesta de Moulines establece una "condición de vinculación de los términos $T$ no-teóricos" (LNT), mediante la cual relaciones o vínculos interteóricos garantizan la existencia de métodos de determinación externos a una teoría $T$, que posibiliten la determinación de algún conjunto de términos en $T$, vale decir, que ayuden a delimitar los términos $T$-no-teóricos relativos a $T$. Uno de los ejemplos más citados es la no-teoreticidad de la función posición en la mecánica newtoniana de partículas, que se asocia al hecho de que la posición se vincula con las funciones de longitud y ángulo de la geometría física y existen métodos geométricos de determinación de la longitud y del ángulo que no presuponen a las leyes de la mecánica newtoniana.

"El objetivo de la condición de $L N T$ es inducir la formulación de la hipótesis metaempírica de que todos los términos no-teóricos de cualquier teoría científica están esencialmente vinculados con algunos términos de otras teorías" (Moulines 1985, p. 477). De cumplirse esta condición, más el criterio $C T^{\prime 8}$ (en verdad se trata de la negación de), tendríamos lo que el autor llama "términos no-teóricos normales". Moulines se pregunta si podría haber términos no-teóricos "anormales" en la ciencia. Éste es un problema significativo y, como hace notar el autor, si todos los términos-no-teóricos en $T$ satisfacen para alguna teoría diferente $T_{i}$ la $L N T$, entonces el sentido de "teórico" podría extenderse y decirse que son teóricos en un sentido derivado. Ello conduciría a que no nos podríamos salir de una especie de macrorred con vínculos interteóricos y perderíamos la posibilidad de hablar de datos empíricos extralingüísticos o extramodélicos; seguidamente perderíamos la posibilidad de pensar una justificación teórica sobre "bases externas" a la teoría en cuestión y deberíamos inclinarnos a favor de un criterio coherentista de justificación en la ciencia.

Sin embargo, parecería que ni en los planteos tradicionales de Sneed y Stegmüller, ni tampoco en las elaboraciones posteriores de Moulines, se esté dispuesto a pensar que la salida al problema sea de tipo coherentista. Al admitir $L N T$ se está exigiendo que exista una teoría y un

${ }^{8}$ Como dice el mismo Moulines, se trata en verdad de la negación del $C T^{\prime}$, o de una versión más sofisticada del mismo: un término es $T$-no-teórico si y sólo si no es $T$-teórico en el sentido de $C T^{\prime}$ (Moulines 1985, p. 477). 
modo de determinación del significado de un $T$-teórico distinto de $T$. De este modo se puede contar con la posibilidad determinar los $M_{p p}$ de una teoría $T$ en cuestión como constituyendo la base empírica de $T$. Así seguiríamos sosteniendo que para que una teoría científica pueda diferenciarse de una teoría ficcional debe existir algún tipo de entidades extralingüísticas de las que podamos dar cuenta sin presuponer en todo momento $T$. Esta manera de hablar de "entidades extralingüísticas" puede ampliarse cuando extendemos $L N T$ como condición a un conjunto de teorías relacionadas entre sí por un conjunto de links, y/o relaciones interteóricas, situación que resulta particularmente clara cuando hablamos de red teórica.

\section{5}

Conviene aquí agregar que el problema planteado respecto a la salida coherentista como consecuencia de la caracterización de "término teórico" en la ciencia ha sido también pensada dentro de la CE. Balzer $(1986,1996)$ ha señalado que el $C T$ es un criterio que distingue los términos teóricos basándose en nociones pragmáticas y, a diferencia de esta propuesta, el autor propone un nuevo criterio de teoreticidad que intenta deslizar el eje de los argumentos acerca de la teoreticidad de los términos científicos hacia un criterio más semántico y formal.

El criterio de Balzer $(1986,1996)$-basado parcialmente en Gähde (1983) - busca una caracterización más exhaustiva que la basada en nociones tales como los "métodos de medición de una función", y brinda lo que el autor denomina una "solución coherentista" al problema. Al mismo tiempo caracteriza la noción de teoreticidad en el marco de lo que llamamos teoría en sentido genérico (red teórica). Balzer parte de la necesidad de caracterizar más clara y formalmente la noción de "teoreticidad" y argumenta que el énfasis que se ha dado a la importancia de la distinción $T$-teórico, $T$-no-teórico, cuyo rol en la metateoría estructural ha sido a su juicio sobredimensionada (particularmente en Stegmüller (1973)), podría deflacionarse de modo que el problema se oriente más específicamente a una definición semántica y formalmente clara de la noción aludida.

Sin embargo, sólo mencionamos aquí la posición de Balzer a los efectos, hacer notar ciertas no-homogeneidades en el tratamiento del tópico dentro de la CE, ya que nuestro interés, tal como ha sido antes 
comentado, es profundizar las consecuencias ontológicas de la solución pragmática del problema de los términos en la ciencia.

\section{Segunda Parte}

6

Dejando de lado la conceptualización de Balzer nos centraremos en la posición sneediana y moulinesiana, a partir de las cuales haremos notar que:

(a) La diferenciación entre términos $T$-teóricos y términos $T$-noteóricos es central para distinguir los modelos de una teoría de los sistemas físicos, o empíricos, que tienen la posibilidad de "caer bajo" el predicado conjuntista que caracteriza a dicha teoría.

(b) La diferenciación anterior nos permite determinar que, dada una teoría $T$ en cuestión, $T$ no conforma el sistema "a piachere", sino a la forma de $T$, pero lo importante aquí es que se presupone la existencia de alguna $T^{\prime}$ diferente de $T$ que puede conceptualizar de un modo independiente de (y no incompatible con) $T$ algún sistema físico o empírico (condición de $L N T$ ).

(c) A partir de lo anterior afirmaremos que esta caracterización nos aleja de una posición irrealista (la teoría no crea los sistemas, no hay tantos sistemas posibles como teorías posibles; los sistemas no son creados por la teoría, sino que se conforman a ella). Podríamos agregar que esta afirmación tiene consecuencias en relación con la idea de unificación de la ciencia por un lado, y por el otro, con alguna concepción -no precisada- de "unicidad del mundo".

Si bien la propuesta pragmática del problema de la determinación de los términos en la ciencia dentro de la CE no parece compatible con una posición irrealista, parecería que sí puede serlo tanto con una posición antirrealista como con una realista.

Los trabajos sobre la ontosemántica de la ciencia, presentados particularmente por Moulines (1984, 1994, 1998, 2002), se inscriben, a mi entender, en una perspectiva que podemos estimar como antirrealista. En Moulines (2002) el autor nos propone que la pregunta esquemática 
fundamental: (1) ¿Hay esto? Debe ir precedida por (2) ¿Cómo puedo saber que hay esto? Pregunta que a su vez depende de la respuesta que demos a (3) ¿Qué significa "esto"?

Moulines (2001) estima que el primer filósofo en haberse percatado plenamente de la mutua imbricación de las preguntas (1), (2) y (3), así como de la preeminencia metodológica de (3), fue Gottlob Frege y [por ello] podemos considerarlo como el pionero avant la lettre de la disciplina que Moulines llamó 'ontoepistemosemántica'. A continuación agrega: "La semántica que tengo en mente, y que es componente fundamental de la ontoepistemosemántica, es una semántica que engloba la pragmática, al menos en la medida en que esta última sea relevante para las preguntas [1, 2 y 3$]$ anteriores. Precisamente concibo lo que se puede llamar el aspecto no-referencial del significado de las expresiones como esencialmente pragmático" (Moulines 2002, pp. $1-2)$.

Podemos comprender esto en el marco de las ideas trazadas del planteamiento sneediano: los procesos de construcción de las teorías científicas se desarrollan conforme a ciertos patrones de la comunidad científica que fijan dinámicamente los significados conceptuales, pero estos procesos no son concebidos como causales, sino selectivos en cuanto se hallan guiados por intenciones de los científicos.

Moulines nos dice además que "la determinación del significado depende en parte, Wittgenstein dixit, de los usos, o sea, de la pragmática de los términos de las teorías" ...(Moulines 2002, p. 4). En efecto, el autor sostiene: "Podemos (y debemos, según creo) colorear la semántica básicamente fregeana con un matiz wittgensteineano. Wittgenstein nos enseñó que (en una gran clase de casos) 'el significado de una palabra es su uso en el lenguaje”"9 (Moulines 2002, p. 1). Explicitando su posición Moulines afirma:

Mi punto de partida es fregeano - modificado por Tarski (para el aspecto referencial) y por Wittgenstein (para el aspecto no-referencial). Si entendemos por "semántica" la teoría general del significado (como yo propondría), entonces Frege nos enseñó que el significado de una expresión (sea oración o una parte de una oración, para este contexto da igual) es la resultante de dos 'vectores': referencia y sentido. El primer vector es puramente semántico, el segundo parcialmente pragmático. El valor de verdad

${ }^{9}$ Cfr. L. Wittgenstein, Investigaciones filosoficas, Parte I, §41. Citado por Moulines (2002, p. 2). 
del enunciado 'hace 70 millones de años se paseaba un dinosaurio por el Englischer Garten de Munich' es V o F con independencia de toda consideración pragmática (y aunque nadie haya usado, use o vaya a usar jamás esa oración); asimismo, los modelos de un sistema axiomático (como conjunto de fórmulas) vienen determinados a lo Tarski, sin que la pragmática pinte nada (al menos en un sentido no-trivial de "pragmática"). Ahora bien, Frege también nos enseñó que la vía de acceso de una expresión a su referencia, su sentido, también forma parte esencial del significado de una expresión, y lo que yo afirmo es que en esa vía de acceso tienen un importante papel ciertos parámetros claramente (y no-trivialmente) pragmáticos. Esos son los "usos" de Wittgenstein y cosas parecidas. ${ }^{10}$

Los que estamos familiarizados con la $\mathrm{CE}$ recibimos como accesible el acercamiento entre la CE y la noción fregeana de referencia, pero a algunos les cuesta comprender la idea de "aspectos no-referenciales del significado" asociada a la idea wittgensteniana de "uso", aún más cuando se afirma: "Estos usos son (parte de) los 'sentidos' de los términos según el significado originario que Frege dio a 'sentido'" (Moulines 2002, p. 3).

Si comprendo bien, "el modo de darse la referencia" (el sentido) vendría a darse pragmáticamente (aunque sólo en parte). Si fuese así, y tomando en cuenta que los otros aspectos de la determinación del significado que quedan excluidos de la pragmática, supongo son aspectos que caen habitualmente en la semántica, mi cuestionamiento sería: ¿cuáles son? Queda claro para mí que no se quiere circunscribir la cuestión del significado al tratamiento semántico, pero no se vislumbra si se está dispuesto a subsumir ésta en la pragmática.

A este interrogante ha respondido Moulines:

Si entendemos por "semántica" la teoría general del significado, si admitimos con Frege que significado $(x)=\langle\operatorname{referencia}(x)$, sentido $(x)\rangle$ y si le damos un toque wittgensteineano a ese $\operatorname{sentido}(x)$ resulta que "la semántica engloba la pragmática". Ahora bien, no creo que todos los aspectos intensionales de una expresión (el sentido) puedan (o deban) definirse en términos estrictamente pragmáticos, están, por ejemplo, las modalidades, los conjuntos de mundos posibles admitidos para esa expresión y cosas parecidas, ello se puede tratar de manera estrictamente semántica ("semántica de mundos posibles"). Estos aspectos no deben ser olvidados en un tratamiento general del significado, pero son no-referenciales (en mi sentido y,

${ }^{10}$ Texto inédito que forma parte de comunicaciones escritas mantenidas con el autor (abril/junio 2002). 
creo, en el de Frege). Si tu pregunta era: '¿No-referencial = pragmático?' la respuesta es claramente: 'no'. ${ }^{11}$

En Pluralidad y recursión Moulines había ya indagado sistemáticamente la distinción global entre sentido y referencia. La lectura del texto me sugiere la idea de que hay una identificación entre la dupla sentido/referencia y la dicotomía aspectos no-referenciales del significado/referencia, ya que el "sentido" de Frege se interpreta en parte pragmáticamente (a lo Wittgenstein) y en parte semánticamente (incluyendo los aspectos modales y otros) y, por ende, es una expresión que engloba todos los aspectos del significado no incluidos en la referencia.

A los comentarios previos Moulines responde:

Lo que me parece más importante del modo como Frege caracteriza el sentido es que sea "la vía de acceso" de la expresión a su referente, y si ahora nos preguntamos en qué consiste esa "vía de acceso", creo que la respuesta es que ello variará enormemente según los tipos de discurso y según el tipo de expresiones dentro de cada tipo de discurso. Mi interés primordial está en el sentido de los términos teóricos primitivos en las ciencias empíricas establecidas, en ese contexto, dentro del sentido de los términos caben, según creo, diversas cosas y entre ellas algo así como los "usos" wittgensteineanos -ya sea sólo porque para determinar el referente de, pongamos por caso, el término "masa" hay que hacer uso de una balanza que ha de funcionar de acuerdo con ciertas reglas "negociadas" por la scientific community - y aquí nos salta a la cara la pragmática. [...] Ahora bien, esta matización del sentido fregeano con componentes pragmáticos no significa que yo esté abogando por una reducción a la pragmática del sentido ni mucho menos de la semántica en general. ${ }^{12}$

Como se ve, la propuesta moulinesiana nos advierte que los aspectos no referenciales del significado de un término no pueden identificarse sin más con la noción de "sentido" fregeano; sino que trata más bien de ampliar la semántica fregeana a campos pragmáticos, para lo cual la noción wittgensteiniana de "uso" le parece al autor una vía prometedora, aunque como comentaré luego, esta salida no me parece fácil de llevar a cabo.

${ }^{11}$ Véase la nota 10.

${ }^{12}$ Ibídem. 


\section{Conclusiones}

En los puntos anteriores se ha pretendido esbozar un conjunto de problemas ontosemánticos en el marco de la CE, centrándonos en el problema de la distinción entre términos $T$-teóricos y $T$-no-teóricos, y las consecuencias ontológicas de esta distinción.

Hemos distinguido entre una solución pragmática y una semánticoformal al problema, atribuyendo a los enfoques de Sneed, Stegmüller y Moulines la caracterización de pragmáticos, y al de Balzer la de semántico-formal. Hecho esto nos hemos separado del enfoque de Balzer (1986, 1996) para concentrarnos en la primera solución.

En relación a la propuesta de Sneed (1971) hemos señalado las nociones pragmáticas involucradas en la distinción aludida. En relación con éstas, hemos comentado el rol central de las nociones de "determinación" y "métodos de determinación" de los términos teóricos, así como las nociones de "presuposición" y de "disponer de una teoría". De este modo, hemos enfatizado el rol de la concepción de "intended application", que implica la idea de que ciertas estructuras se aplican a cierto dominio empírico de acuerdo a una concepción pragmáticointencional de aplicación: se consideran aplicaciones paradigmáticas, esto es, un subconjunto del conjunto total de aplicaciones, dado en un momento del desarrollo teórico, que la comunidad científica reconoce como paradigmático. En esta última idea se remarca el rol de los componentes sociológicos e históricos que intervienen en la configuración de las aplicaciones intencionales. Finalmente, respecto de este aspecto, la relación entre la estructura formal y el conjunto de aplicaciones intencionales se presenta con un carácter instrumental y convencional: nuestras construcciones modeloteóricas pueden servirnos para la descripción y explicación de cierto sistema fenoménico al que se aplica, según objetivos de la comunidad científica en circunstancias históricopragmáticas determinadas.

A pesar de sus aciertos, el CT de Sneed resultaba demasiado restringido y merecedor de diversas críticas. En Balzer (1996) se remarcan algunos problemas que podrían devenir del criterio:

Con respecto a la presencia de términos teóricos la cuestión es si se asume que ellos ocurren en todas las aplicaciones intencionales del modelo metateórico. En su formulación original Sneed no asumió que todas las teorías 
contienen términos empíricos, su modelo da espacio para casos extremos donde todos o ninguno de los términos son T-teóricos”. (Balzer 1996, p. 153)

Stegmüller (1973, 1976) mostraba un claro interés filosófico por profundizar y desarrollar las implicaciones del enfoque pragmático presente en $C T$, tanto en relación a los aspectos epistémicos, como en los actitudinales en la ciencia y la actividad científica. Sus reflexiones señalaban la necesidad de elucidar nociones tales como "alguien" "dispone" de una teoría; o "alguien" "sabe" o "cree" que $Y$ en el contexto $t$. Sin embargo, a pesar de su interés manifiesto en la elucidación metateórica aquellas nociones permanecieron sin un tratamiento posterior más exhaustivo.

A partir de Balzer y Moulines (1980) la ampliación del marco metateórico permitiría mayor precisión para el tratamiento de la distinción entre términos $T$-teóricos y $T$-no-teóricos. La posición seguida luego en Moulines (1985) representó una profundización y clarificación de la propuesta original de Sneed y Stegmüller. La $L N T$ constituyó un requisito importante en la determinación de no-teoreticidad, constituyendo un modo de escapar a la demasiado amplia concepción hansoniana o achinsteiniana de la "carga teórica" de los términos científicos. Asimismo, en la propuesta de Moulines la distinción entre axiomas y leyes fundamentales de la teoría, por un lado, y vínculos o links interteóricos (principios puente) por otro, sirvió de base conceptual a la distinción de términos $T$-teóricos y $T$-no-teóricos, a la vez que aportó una herramienta importante para la reconstrucción modeloteórica de las teorías científicas.

En referencia a la relación entre la distinción de términos en la ciencia y la vía de acceso a la pregunta ontológica hemos considerado que la CE no resulta compatible con un irrealismo, pero podría serlo tanto con una posición realista como con una antirrealista.

En nuestro análisis hemos examinado la posición moulinesiana, que englobamos en una concepción antirealista, dada su tesis de la determinación primaria del lenguaje en el modo de acceder a los entes en general y a las entidades científicas en particular. ${ }^{13}$

${ }^{13}$ Algunos otros podrían también pensar que la posición moulinesiana es conciliable con el realismo interno de Putnam (1994), quien sostiene que "se debe abandonar la dicotomía tradicional entre el mundo "en sí mismo" y los conceptos que usamos para pensar y hablar 
Por su parte la postura moulinesiana respecto de la determinación del significado de los términos científicos presenta un acercamiento de la pragmática wittgensteiniana. Como vimos, en la interpretación propuesta por el autor se asume que a pesar de que la concepción fregeana de referencia está en la base de la concepción semántica de las teorías científicas, es necesario reconocer aspectos no-referenciales del significado.

Sin embargo creo que la interpretación wittgensteiniana de "sentido" fregeano, aportada por Moulines, resulta difícil de sostener. En una línea de interpretación de Frege ${ }^{14}$ se afirma que podemos concebir el sentido fregeano en términos de conocimiento compartido, que nos permite la identificación del referente. Así, cuando Frege sostiene que "el sentido es el baluarte más preciado de la humanidad", una interpretación bastante generalizada, asocia "sentido" con "saber qué”, que en Frege se relaciona con la idea de saber objetivo, lógico (Frege 1892, 1918). Pero una lectura pragmática implicaría una interpretación de "sentido" ligada a la idea de "saber cómo", lo cual es ciertamente difícil de sustentar con base en los textos fregeanos. Tal vez se podría optar por una conceptualización tripartita del significado, sumando a la categorización fregeana algún término nuevo, o usar otro término con mayor abarque, que incluya "sentido" fregeano, pero agregue el componente pragmático. Lo que me parece es que el "sentido" fregeano no puede interpretarse pragmáticamente sin que se convierta en algo no-fregeano.

Creo que pensar los $M_{p p}$ de una teoría $T$ en términos de las entidades que pueden ser definidas o caracterizadas por algún conjunto que contenga al menos una teoría distinta de $T$ es en parte un modo original de hablar de la ontología de la teorías, pero al mismo tiempo un modo tradicional de hacerlo. Es un modo original en el sentido de que rompe con la idea de base empírica concebida como base observacional, dejando afuera el complejo y debatido problema de las entidades y términos observacionales. La originalidad se marca también en los componentes pragmáticos incorporados al criterio de teoreti-

sobre él, y se afirma que nuestras entidades mundanas y objetos cognitivos son siempre teórico-dependientes".

${ }^{14}$ En ésta se ubicaría claramente la posición de Dummett (1990), por ejemplo. 
cidad, especialmente en los relativos al rol de la idea de aplicaciones intencionales (y en particular, aplicaciones paradigmáticas).

Sin embargo la propuesta resulta tradicional en el sentido de que la disponibilidad de una teoría se piensa en relación a la posibilidad de hacer aserciones teóricas acerca de $X$, donde $X$ debe amoldarse (modelarse) según $T$, (aunque al mismo tiempo debe poder ser de algún modo modelable en un lenguaje diferente de $T$ ). Disponer de una teoría $T$ está por ende asociado a la idea de realizar aserciones empíricas de T. Pensando en otros desarrollos (Hacking 1996, 2003, entre otros), la caracterización de pragmática de esta disponibilidad de $T$ se halla aún bastante circunscripta a una imagen de la ciencia como actividad asertórico veritativo-funcional.

Asimismo, estimo que la solución pragmática a que hemos aludido arriba fue programáticamente prometedora. Tanto la concepción originaria de Sneed como la de Stegmüller sobre los términos científicos contenían un conjunto de nociones pragmáticas, entendiendo por tales nociones relativas a las prácticas científicas. Éstas permitieron un diálogo fecundo entre la CE y las ideas de Kuhn de ciencia normal, comunidad científica, etc., como también ocurrió con la concepción de Hempel (1973). Pero el camino posterior de la CE -aún a pesar de los interesantes aportes metateóricos de Moulines- siguió una vía de clarificación metateórica, de aumento de la formalización que, si bien ayudó a ganar en precisión, fue lentamente dejando sin profundizar las nociones pragmáticas programáticamente introducidas en los comienzos.

Finalmente, cabe agregar a la propuesta moulinesiana de extender la noción de "sentido" fregeano, Wittgenstein dixit, a una pragmática es sin duda mucho menos abarcadora que la propuesta original antes referida. Esta salida se circunscribe al campo de la pragmática del significado, pero deja de lado muchos otros aspectos que serían parte de una agenda de problemas del enfoque pragmático sobre la determinación de los términos en la ciencia, rasgos que han sido ubicados en una dimensión praxista del análisis de la ciencia que incluye aspectos sobre modos, tipos y rasgos de las acciones en las prácticas científicas, aspectos valorativos -no sólo epistémicos- y prácticas regladas sobre conductas normativas. Aunque el catálogo de problemas podría seguirse, los aspectos pragmáticos de la ciencia ligados a la determinación de los 
términos científicos en las vías de tratamiento de la CE han quedado muy circunscritos a una esfera ontosemántica, u ontoepistemosemántica como Moulines prefiere denominarla, cuyos planteos, a pesar de las aristas restrictivas expuestas, constituyen los aportes contemporáneos más significativos al tratamiento del problema en el marco de la tradición estructuralista de la ciencia.

\section{Referencias}

Achinstein, P., 1989, “Conceptos de la ciencia”, en Olivé y Perez Ransanz 1989, pp. 355-381.

Balzer, W., 1986, "Theoretical Terms, A New Perspective", The Journal of Philosophy, no. 83, pp. 71-90.

—, 1996, "Theorical Terms: Recent Developments", en Balzer y Moulines 1996, cap. 8.

Balzer, W. y Moulines, U., 1980, "On Theoreticity", Synthese, vol. 44 no. 3, pp. 467-494.

- (comps.), 1996, Structuralist Theory of Science, Walter de Gruyter, Berlín.

Balzer, W., C.U. Moulines y J.D. Sneed, 1987, An Architectonic for Science. D. Reidel, Dordrecht.

Balzer, W., D. Pearce y H.J. Schmidt (eds.), 1984, Reduction in Science, D. Reidel, Dordrecht.

Balzer, W. y J.D. Sneed, 1977, "Generalized Net Structures in Empirical Sciences I”, Studia Logica, no. 36 , pp. 195-211.

— 1978, "Generalized Net Structures in Empirical Sciences II", Studia Logica, no. 37, pp. 167-194.

Dummett, M., 1990, "La filosofía de Frege", en Dummett, 1990, pp. 157-189.

—, 1990, La verdad y otros enigmas, Fondo de Cultura Económica, México.

Frege, G., 1892, "Über Sinn und Bedeutung", Zetschrift für Philosophie und philosophische Kritik, C, pp. 25-50. Versión utilizada: "Sobre sentido y referencia" en Estudios sobre Semántica, Ariel, Barcelona, 1984, pp. 51-86.

—, 1918, "Der Gedanke. Eine logische Untersuchung", Beiträge zur Philosophie des deutschen Idealismus, vol. 1, pp. 58-77. Versión utilizada: "El pensamiento: Una investigación lógica, en Investigaciones Lógicas, Tecnos, Madrid, 1984, pp. 49-85.

Gähde, U., 1983, T-Theoretizität und Holismus, Peter Lang, Francfort del Meno/ Berna.

Gómez Pin, V. (comp.), 1994, Categorías e inteligibilidad global, Universidad Autónoma de Barcelona, Barcelona.

Hanson, N. R., 1958, Observación y explicación: guía de la filosofía de la ciencia. Patrones de descubrimiento, Alianza Editorial, Madrid. 
Hempel, C., 1973, "El significado de los términos teóricos: una crítica de la concepción empirista estándar”, en Olivé y Ransanz 1989, pp. 439-453.

- 1990. "On Intertheoretical Conditions for Theoretical Terms", Erkenntnis, vol. 32, pp. 215-233.

Hacking, I., 1983, Representing and Intervening: Introductory Topics in the Philosophy and Natural Science Cambridge University Press, Cambridge.

—, 1993, "Working in a New World: The taxonomic Solution", en World Changes: Thomas Kuhn and the Nature of Science. Horwick, P. (ed.) Cambridge, Mass.: MIT Press, 275, 310.

Kamlah, A., 1986, "An Improved Definition of 'Theoretical in a Given Theory' ", Erkenntnis, vol. 10, pp. 349-359.

Kuhn, T., 1962, La Estructura de las revoluciones científicas, Fondo de Cultura Economica, México.

—, 1976, "Theory Changes as Structure Change: Remarks on the Sneed Formalism", Erkenntnis, vol. 10, pp. 179-199.

Moulines, C.U., 1982, Exploraciones metacientíficas, Alianza Editorial, Madrid.

— , 1984, "Ontological Reduction in the Natural Sciences", en Balzer, Pearce y Schmidt 1984.

— 1985, "Theoretical Terms and Bridge Pinciples: A Critique of Hempel's (Self-) Criticism", Erkenntnis, vol. 22, pp. 97-117. Versión utilizada: "Los términos teóricos y los principios puente: una crítica de la (auto)crítica de Hempel”, Filosofía de la ciencia: teoría y observación, en Olivé y Perez Ransanz 1989, pp.

—, 1991, Pluralidad y recursion. Estudios epistemológicos, Alianza Editorial, Madrid.

—, 1994, “¿Qué clases de cosas hay?”, en Gómez Pin 1994, pp. 25-33.

—, 1998, "Esbozo de ontoepistemosemántica", Theoria, vol. 13, no. 1, pp. $141-159$.

— 2010, "Ontoepistemosemántica en perspectiva estructuralista", en PerisViñé 2010, pp. 17-30.

Olivé y Ransanz (comps.), 1989, Filosofía de la ciencia: teoría y observación, Siglo XXI, México.

Olivé, L. y A.R. Pérez Ransanz (comps.), 1989, Teoría y observación, UNAM, México.

Peris-Viñé, L.M. (comp.), 2010, Filosofía de la ciencia en Iberoamérica: Metateoría Estructural, Tecnos, Madrid.

Putnam, H., 1962, "Lo que las teorías no son", en Olivé y Ransanz 1989, pp. 312-319.

Rolleri, J.L., 1986, Estructura y desarrollo de las teorías científicas, UNAM, México. Sneed, J., 1971, The Logical Structure of Mathematical Physics, D. Reidel, Dordrecht.

- 1976, "Philosophical Problems in the Empirical Science of Science: A Formal Approach”, Erkenntnis, vol. 10, pp. 115-146. 
Stegmüller, W., 1973, Probleme und Resultate der Wissenschaftstheorie und Analytischen Philosophie vol. 1: Theorie and Erfahrung; volumen 2: Theorienstrukturen und Theoriendynamik, Springer, Heidelberg.

_- 1976, "Accidental ('Non Substantial') Theory Change and Theory Dislodgement: to What Extent Logic Can Contribute to a Better Understanding of Certain Phenomena in Dynamics of Theories", Erkenntnis, vol. 10, 1976. Versión utilizada: "Cambio teórico accidental (no sustancial) y desplazamiento de teorías”, en Rolleri 1986, pp. 215-250.

Toumela, R., 1973, Theoretical Concepts, Springer, Viena-Nueva York.

Recibido el 8 de septiembre de 2011 Aceptado el 30 de noviembre de 2011 